\title{
Structural and functional insights into the Diabrotica virgifera virgifera ATP-binding cassette transporter gene family
}

\author{
Folukemi Adedipe ${ }^{1}$, Nathaniel Grubbs', Brad Coates², Brian Wiegmman¹ and Marcé Lorenzen ${ }^{1 *}$
}

\begin{abstract}
Background: The western corn rootworm, Diabrotica virgifera virgifera, is a pervasive pest of maize in North America and Europe, which has adapted to current pest management strategies. In advance of an assembled and annotated D. v. virgifera genome, we developed transcriptomic resources to use in identifying candidate genes likely to be involved in the evolution of resistance, starting with members of the ATP-binding cassette (ABC) transporter family.

Results: In this study, 65 putative D. v. virgifera $A B C$ (DvVABC) transporters were identified within a combined transcriptome assembly generated from embryonic, larval, adult male, and adult female RNA-sequence libraries. Phylogenetic analysis placed the deduced amino-acid sequences of the DVABC transporters into eight subfamilies (A to H). To supplement our sequence data with functional analysis, we identified orthologs of Tribolium castaneum $A B C$ genes which had previously been shown to exhibit overt RNA interference (RNAi) phenotypes. We identified eight such $D$. v. virgifera genes, and found that they were functionally similar to their $T$. castaneum counterparts. Interestingly, depletion of DVVABCB_39715 and DVVABCG_3712 transcripts in adult females produced detrimental reproductive and developmental phenotypes, demonstrating the potential of these genes as targets for RNAimediated insect control tactics.
\end{abstract}

Conclusions: By combining sequence data from four libraries covering three distinct life stages, we have produced a relatively comprehensive de novo transcriptome assembly for D. v. virgifera. Moreover, we have identified 65 members of the $A B C$ transporter family and provided the first insights into the developmental and physiological roles of $A B C$ transporters in this pest species.

Keywords: ATP-binding cassette (ABC) transporter, Phylogenetic, Transcriptome, RNA interference (RNAi), Corn rootworm

\section{Background}

The western corn rootworm, Diabrotica virgifera virgifera (Coleoptera: Chrysomelidae), is a major pest of maize in Europe and North America [1-3], where costs of management, as well as crop losses attributed to damage by this pest, are estimated at over 1 billion U.S. dollars annually in North America alone (reviewed in [4]). The notorious difficulty facing efforts to control D. v. virgifera feeding on maize has arisen via intra-species adaptations that overcome various pest management methods [5]. For example,

\footnotetext{
*Correspondence: marce_lorenzen@ncsu.edu

'Department of Entomology and Plant Pathology, North Carolina State University, Box 7613, 1566 Thomas Hall, Raleigh, NC 27695-7613, USA Full list of author information is available at the end of the article
}

changes in oviposition preference within "soybean variant" populations of D. v. virgifera in the Midwest United States circumvent the cultural-control practice of corn-soybean rotation [3, 5-7]. Additionally, adapted phenotypes within North American D. v. virgifera populations can survive high exposures to organochlorine [8], pyrethroid [9], and carbamate and organophosphate insecticides [10]. In some instances resistant phenotypes have persisted for decades despite the removal of selection pressures [11]. More recently, field populations of $D . v$ virgifera have developed high levels of resistance to transgenic maize hybrids that express Bacillus thuringiensis (Bt) crystal toxins Cry3Bb1 [12], mCry3A [13], Cry3.1Ab [14], and Cry34/35Ab1 [14-17]. However, RNA interference (RNAi) shows great potential

(c) The Author(s). 2019 Open Access This article is distributed under the terms of the Creative Commons Attribution 4.0 International License (http://creativecommons.org/licenses/by/4.0/), which permits unrestricted use, distribution, and 
as a novel insect pest control technology [18], especially in instances where target species are sensitive to oral RNAi [19]. D. v. virgifera is highly sensitive to oral RNAi [20-22], suggesting that it could suppress feeding damage caused by this pest [23].

ATP-binding cassette $(\mathrm{ABC})$ proteins comprise one of the largest gene families, and are found across prokaryotic and eukaryotic domains [24]. Most of these proteins function as transmembrane transporters, which actively move a myriad of molecules across cellular membranes [25]. ABC transporter proteins have a two-domain structure: a highly conserved nucleotide-binding domain (NBD) and a variable transmembrane domain (TMD) [26]. The NBD binds and hydrolyzes ATP to provide the energy required for translocating a substrate across cell membranes, while the TMD forms a channel through which the substrate is transported [27]. Each NBD possesses several highly conserved, characteristic motifs, including Walker A, Walker B, Q-loop, D-loop, H-loop, and $A B C$ signature motifs, while each TMD is made up of five to six transmembrane $\alpha$-helices that dictate substrate specificities [27]. ABC transporter proteins require two NBDs and two TMDs for functionality. Some ABC transporters are full-transporters (FT) in that two TMDs and two NBDs are encoded in a single protein, whereas most are half-transporters (HT; one TMD and one NBD) and form functional units following homodimerization or heterodimerization [24, 27, 28]. Due to the relatively conserved sequence of the $\mathrm{NBD}$, it has been used for the phylogenetic classification of the $A B C$ transporter superfamily into eight subfamilies designated A to $\mathrm{H}$ (ABCA to $\mathrm{ABCH})$ [29].

Among insect species, $\mathrm{ABC}$ transporters are implicated in diverse functions, including transportation of eye pigments [30-34], and resistance to chemical insecticides $[35,36]$. Within the model species for Coleoptera, the red flour beetle, Tribolium castaneum, Broehan et al. [30] reported that RNAi-mediated knockdown of some ABC transporters resulted in mortality, or phenotypes characterized by arrested growth, abnormal cuticle formation, defective eye pigmentation, or abnormal egg-laying or -hatching. Changes in the expression level or structure of some ABCA, ABCC and ABCG subfamily members have been associated with Bt toxin resistance in species of Lepidoptera [37], while paralogs of an ABCB transporter were linked to Bt Cry3Aa resistance in the coleopteran species, Chrysomela tremula [38], and were found to be in proximity to a quantitative trait locus (QTL) for Cry3Bb1 resistance in D. v. virgifera [39].

Similar investigations of $\mathrm{ABC}$ transporters in $D . v$. virgifera are arguably limited due to the dearth of genomic resources available for this species, which are currently comprised of Sanger and Roche 454 read-based transcriptome assemblies [40-43]. Complicating the development of genomic tools is the $2.58 \mathrm{~GB}$ size and complex repetitive structure of the D. v. virgifera genome [4, 44]. Regardless, RNA sequencing (RNA-seq) has become an expeditious and cost-effective method for obtaining a wealth of transcriptome sequence data in non-model insects [45]. In the following, a de novo transcriptome assembly approach was used for the first prediction, annotation, and functional analysis of the $\mathrm{ABC}$ transporter gene family in $D . v$. virgifera. Specifically, eight $A B C$ transporters were identified as putative orthologs to those previously reported to have a defining RNAi phenotype in the model coleopteran species, T. castaneum [30] (DvvABCA 50718, DvvABCB_39715, DvvABCE_2830, DvvABCF 2701, DvvABCG_3712, DvvABCG_14042, Dvvw and DvvABCH_5118). Subsequent RNAi-mediated knockdown demonstrated conservation of function with $T$. castaneum, as well as established potential new insecticidal targets for the control of this devastating agricultural pest.

\section{Results}

Transcriptome sequencing, assembly, and annotation Over 22 million raw Illumina (MiSeq) sequencing reads were generated across four libraries (Table 1; NCBI SRA database accession SRP161473: experiments SRX4669438

Table 1 Paired-end RNA-sequencing libraries and sequencing

\begin{tabular}{|c|c|c|c|c|c|c|c|}
\hline \multirow[b]{2}{*}{$\mathrm{ID}$} & \multirow[b]{2}{*}{ Lib_name } & \multirow[b]{2}{*}{ Insert } & \multirow{2}{*}{$\begin{array}{l}\text { Miseq } \\
\text { Lanes }\end{array}$} & \multicolumn{2}{|c|}{ Raw read data } & \multicolumn{2}{|c|}{ Trimmed read data } \\
\hline & & & & Length & Count & Paired & Unpaired \\
\hline Af1 & DvvAdultF_R1 & 600 to $700-b p$ & 1 & 300-bp & $3,462,470$ & $2,753,272$ & 559,611 \\
\hline Af2 & DvvAdultF_R2 & & & 300 & $3,462,470$ & $2,753,272$ & 72,301 \\
\hline Am1 & DvvAdultM_R1 & 600 to $700-b p$ & 1 & 300 & $2,223,027$ & $1,859,087$ & 308,133 \\
\hline Am2 & DvvAdultM_R2 & & & 300 & $2,223,027$ & $1,859,087$ & 23,335 \\
\hline E1 & DvvEggs_R1 & 600 to $700-b p$ & 1 & 300 & $2,690,038$ & $2,146,192$ & 425,023 \\
\hline E2 & DvvEggs_R2 & & & 300 & $2,690,038$ & $2,146,192$ & 62,149 \\
\hline L1 & DvvLarvae_R1 & 600 to $700-b p$ & 1 & 300 & $2,652,196$ & $2,071,100$ & 445,600 \\
\hline \multirow[t]{2}{*}{ L2 } & DvvLarvae_R2 & & & 300 & $2,652,196$ & $2,071,100$ & 62,923 \\
\hline & & & & Totals & $22,055,462$ & $17,659,302$ & $1,959,075$ \\
\hline
\end{tabular}


to SRX4669441). DNASTAR assembled 13,070,671 reads into a combined transcriptome containing 25,296 contigs with an N50 of $1604 \mathrm{bp}$ (Additional file 1: Table S1). Analogously, assemblies from Trinity and SOAPdenovoTrans respectively produced 162,897 and 133,180 contigs, each with an N50 $\leq 439$ bp (Additional file 1: Table S1). Clustering by CD-HIT-EST reduced complexity 3.5 to $34.9 \%$ across assemblies, and the number of predicted open reading frames (ORFs) within clustered transcripts ranged from 18,305 to 40,087 (Additional file 1: Table S1). BLASTx query of transcripts by Blast2GO against the arthropod-specific section of NCBI's non-redundant $(\mathrm{nr})$ protein database generated annotations for 18,343 DNASTAR contigs ( $E$ value cutoff of $\left.10^{-6}\right)$, with a subset of these receiving gene ontology (GO) mapping and additional annotation terms (Additional file 2: Figure S1). Sequences lacking identity to known arthropod proteins above $E$-value thresholds were attributed to poor sequence conservation and/or novel sequences, as well as non-coding RNAs. The distribution of top BLASTx hits by species showed that $T$. castaneum was the most frequent, representing $65 \%$ of the matches (Additional file 3: Figure S2). Among ontologies assigned via mapping at GO level 2, a majority of the associated terms were assigned to cell structural component, metabolic process, and catalytic activity respectively for GO Cellular Component, Biological Process and Molecular Function (Fig. 1). The DNASTAR assembly showed a high degree of completeness based on a BUSCO score of 928, or $89.6 \%$, of the 1066 genes in the arthropod reference set (v. 9.0) being represented, with analogous levels of representation in both SOAPdenovo-Trans and Trinity assemblies (Additional file 1: Table S1).

\section{Bioinformatic analysis of the $D$. v. virgifera $A B C$ transporter family}

Results of BLASTx queries identified 65 putative D. v. virgifera $\mathrm{ABC}$ transcripts that shared $\geq 37 \%$ amino-acid identity with putative $T$. castaneum orthologs from $\mathrm{ABC}$ transporter subfamilies A through $\mathrm{H}$ (Table 2; Additional file 4: Table S2). Predictions of protein structural domains identified both FTs and HTs. Four DvvABCA and $32 D v v A B C C$ subfamily members were predicted for D. v. virgifera, all of which are FTs. The DvvABCB subfamily contained seven members, which included both full- and half-transporters. The number of assembled $D$. $v$. virgifera paralogs within subfamilies $\mathrm{ABCD}, \mathrm{ABCE}$ and ABCF contained a smaller number compared to DvvABCB, but each had predicted orthologous relationships to T. castaneum ABC transporters. Specifically, the DvvABCD subfamily contained two predicted ABC transporter proteins which were both HTs. One DvvABCE and three $D v v \mathrm{ABCF}$ members were identified, and each of these had two predicted NBD motifs with no TMDs, suggesting that, like their counterparts in other species, they probably do not function as transmembrane transporters. The DvvABCG subfamily contained the second largest number of predicted members with 12, all of which were HTs with only a single NBD and a reverse domain organization. The $D v v A B C H$ subfamily contained four members, which were similar to those of the ABCG subfamily in being HTs with a reverse domain organization. The phylogenetic relationships predicted among NBD regions of deduced $D$. v. virgifera $\mathrm{ABC}$ transporter protein

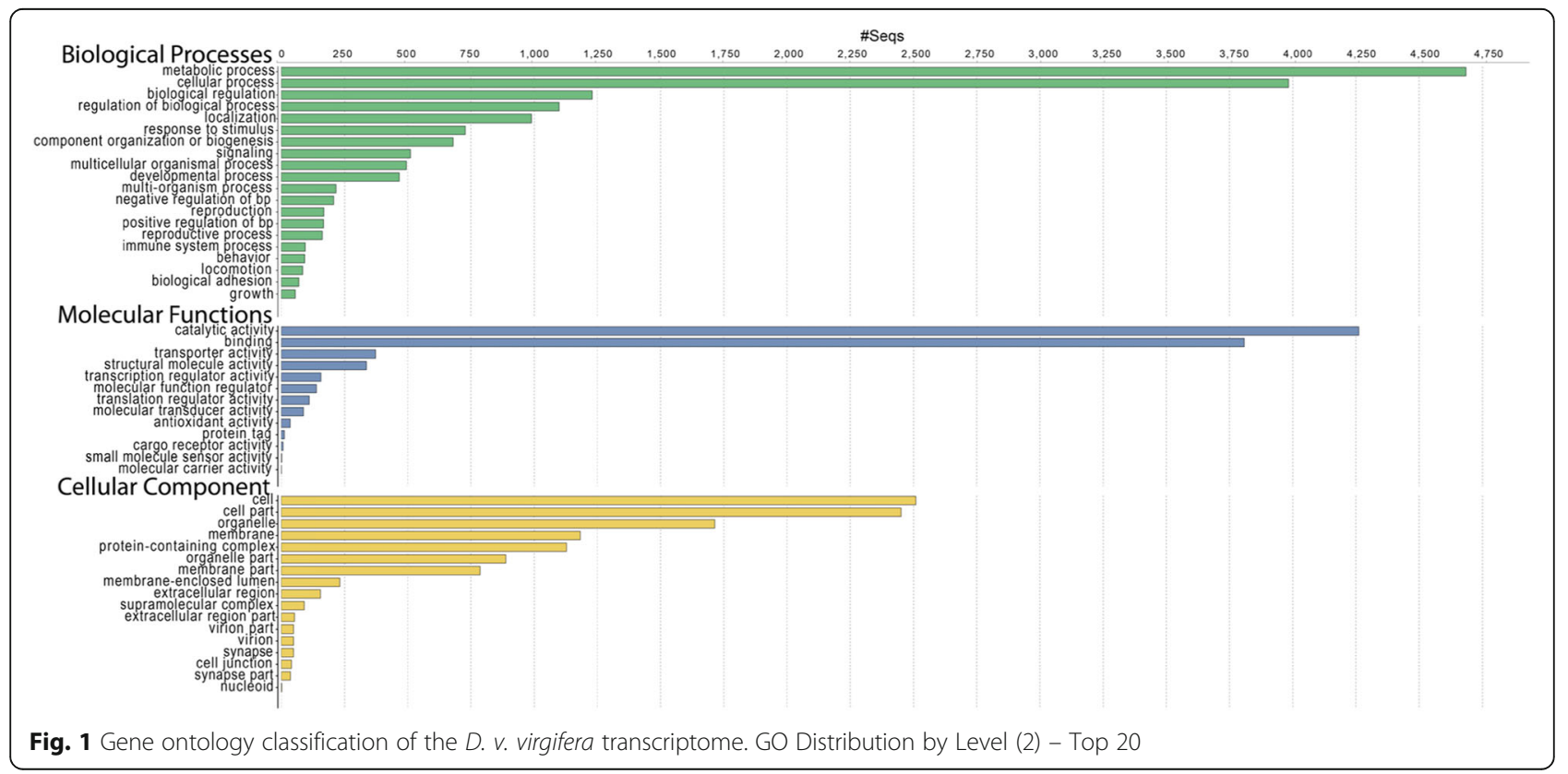


Table 2 Classification of D. v. virgifera ATP binding cassette (ABC) transporters

\begin{tabular}{|c|c|c|c|c|}
\hline \multicolumn{2}{|c|}{ Diabrotica virgifera virgifera transcript } & \multicolumn{3}{|c|}{ Nearest Tribolium castaneum ortholog } \\
\hline Gene ID & Length (aa) & Published Name & Accession & Identity (\%) \\
\hline DvvABCA_18330 & 1756 & TCABCA-UD & XP_008199148.1 & 58 \\
\hline DVvABCA_50718 & 1707 & $T C A B C A-U D^{C}$ & XP_008199148.1 & 56 \\
\hline DvvABCA_49125 & 1643 & TCABCA-7A & XP_008195104.1 & 41 \\
\hline DvvABCA_266167 & 1640 & TCABCA-6A & XP_008195056.1 & 52 \\
\hline DvvABCB_21313 & 1246 & $T C A B C B-3 A$ & XP_00819082.1 & 59 \\
\hline DvvABCB_17742 & 1256 & $T C A B C B-3 B$ & XP_008191266.1 & 63 \\
\hline DvvABCB_19147 & 666 & $T C A B C B-4 A$ & XP_008192744.1 & 72 \\
\hline DvvABCB_39715 b & 715 & $T C A B C B-5 A$ & XP_001813375.1 & 75 \\
\hline DvvABCB_9796 & 833 & $T C A B C B-6 A$ & XP_008194672.1 & 75 \\
\hline DvvABCB_13664a & 657 & $T C A B C B-6 A$ & XP_008194672.1 & 77 \\
\hline DvvABCB_17837 & 681 & $T C A B C B-7 A$ & XP_972133.2 & 69 \\
\hline DvvABCC_41801 & 1267 & $T C A B C C-5 U$ & XP_969849.1 & 36 \\
\hline DvvABCC_44708 & 1256 & $T C A B C C-5 P$ & XP_015836131.1 & 43 \\
\hline DvvABCC_48952 & 1251 & $T C A B C C-5 N$ & XP_971802.2 & 48 \\
\hline DvvABCC_17573 & 1284 & $T C A B C C-5 N$ & XP_971802.2 & 45 \\
\hline DvvABCC_51687 & 1555 & TCABCC-9A & XP_008197311.1 & 71 \\
\hline DvvABCC_21020 & 1296 & TCABCC-5H & XP_968748.1 & 52 \\
\hline DvvABCC_222633 & 1233 & TCABCC-5P & XP_015836131.1 & 46 \\
\hline DvvABCC_18126 & 1342 & TCABCC-5U & XP_969849.1 & 56 \\
\hline DvvABCC_49513 & 1373 & TCABCC-5T & XP_969781.1 & 55 \\
\hline DvvABCC_14070 & 1342 & $T C A B C C-5 U$ & XP_969849.1 & 54 \\
\hline DvvABCC_22628 & 1349 & $T C A B C C-5 R$ & XP_008193834.1 & 55 \\
\hline DvvABCC_20002 & 1344 & $T C A B C C-5 U$ & XP_969849.1 & 56 \\
\hline DvvABCC_7536 & 1363 & $T C A B C C-5 R$ & XP_008193834.1 & 60 \\
\hline DvvABCC_47333 & 1376 & $T C A B C C-5 R$ & XP_008193834.1 & 58 \\
\hline DvvABCC_49618 & 1033 & $T C A B C C-51$ & XP_015835265.1 & 73 \\
\hline DvvABCC_45163 & 1535 & $T C A B C C-4 A$ & XP_008192060.1 & 60 \\
\hline DvvABCC_43960 & 1081 & TCABCC-5H & XP_968748.1 & 49 \\
\hline DvvABCC_48940 & 1223 & TCABCC-5Q & XP_015836083.1 & 43 \\
\hline DvvABCC_217405 & 1164 & TCABCC-5H & XP_968748.1 & 52 \\
\hline DvvABCC_10132 & 870 & $T C A B C C-5 B$ & XP_973693.2 & 55 \\
\hline DvvABCC_48300 & 1257 & TCABCC-5P & XP_015836131.1 & 47 \\
\hline DvvABCC_47673 & 1323 & TCABCC-5T & XP_969781.1 & 71 \\
\hline DvvABCC_5345 & 1257 & TCABCC-5N & XP_971802.2 & 43 \\
\hline DvvABCC_22413 & 1330 & TCABCC-5T & XP_969781.1 & 54 \\
\hline DVvABCC_ $18709^{\mathrm{a}}$ & 1259 & $T C A B C C-5 T$ & XP_969781.1 & 63 \\
\hline DvvABCC_21941 & 1328 & $T C A B C C-5 R$ & XP_008193834.1 & 55 \\
\hline DvvABCC_15305 & 1323 & $T C A B C C-5 T$ & XP_969781.1 & 63 \\
\hline DvvABCC_12562 & 1319 & TCABCC-5H & XP_968748.1 & 53 \\
\hline DvvABCC_10642 & 1306 & $T C A B C C-7 B$ & XP_972534.1 & 63 \\
\hline DvvABCC_41602 & 1307 & $T C A B C C-5 H$ & XP_968748.1 & 54 \\
\hline DvvABCC_12703 & 1317 & TCABCC-5H & XP_968748.1 & 55 \\
\hline DvvABCC_14968 & 1309 & $T C A B C C-5 H$ & XP_968748.1 & 56 \\
\hline
\end{tabular}


Table 2 Classification of D. v. virgifera ATP binding cassette (ABC) transporters (Continued)

\begin{tabular}{|c|c|c|c|c|}
\hline \multicolumn{2}{|c|}{ Diabrotica virgifera virgifera transcript } & \multicolumn{3}{|c|}{ Nearest Tribolium castaneum ortholog } \\
\hline Gene ID & Length (aa) & Published Name & Accession & Identity (\%) \\
\hline DvvABCD_11014 & 754 & $T C A B C D-6 A$ & XP_971218.1 & 75 \\
\hline DvvABCD_11628 & 657 & $T C A B C D-9 A$ & XP_015838765.1 & 80 \\
\hline DvvABCE_2830 & 608 & TCABCE-3A & XP_968009.1 & 91 \\
\hline DvvABCF_2701 ${ }^{\mathrm{b}}$ & 921 & TCABCF-2A & XP_971562.1 & 90 \\
\hline Dvv BCF_802 & 623 & TCABCF-5A & XP_966990.1 & 92 \\
\hline DvvABCF_9935 & 710 & TCABCF-9A & XP_972814.1 & 83 \\
\hline DvvABCG_9811 & 659 & $T C A B C G-4 A$ & XP_008192053.1 & 68 \\
\hline DVvABCG_3712 $2^{\mathrm{b}}$ & 667 & TCABCG-4C & XP_001813184.1 & 77 \\
\hline DvvABCG_14042 ${ }^{\mathrm{b}}$ & 719 & $T C A B C G-4 D^{c}$ & XP_973458.1 & 76 \\
\hline DvvABCG_10897 & 651 & $T C A B C G-4 G$ & XP_008192849.1 & 62 \\
\hline DvvABCG_22358 & 640 & $T C A B C G-4 B$ & XP_015834971.1 & 62 \\
\hline DvvABCG_23081 & 603 & $T C A B C G-4 F$ & XP_971735.1 & 53 \\
\hline DvvABCG_13051 & 637 & $T C A B C G-4 E$ & KYB28165.1 & 60 \\
\hline DvvABCG_38769 & 621 & $T C A B C G-4 H$ & XP_973526.1 & 53 \\
\hline DvvABCG Dvrw ${ }^{b}$ & 657 & TCW & NP_001034521.1 & 60 \\
\hline DvvABCG_49457 & 940 & $T C A B C G-9 C^{c}$ & XP_968472.1 & 73 \\
\hline DvvABCG_36869 & 642 & $T C A B C G-9 D$ & XP_968555.2 & 71 \\
\hline DvvABCG_79525 & 651 & Tcst & NP_001306193.1 & 63 \\
\hline DvvABCH_20789 & 713 & $T C A B C H-9 A$ & XP_973444.1 & 55 \\
\hline DvvABCH_5118 & 795 & TCABCH-9C & XP_008198312.1 & 83 \\
\hline DvvABCH_18290 & 703 & TCABCH-9A & XP_973444.1 & 43 \\
\hline DvvABCH_11818 & 762 & $T C A B C H-9 B$ & XP_967359.1 & 71 \\
\hline
\end{tabular}

${ }^{\mathrm{a}}$ Incomplete sequences, ${ }^{\mathrm{b}}$ RNAi targets, ${ }^{\mathrm{C}}$ not ortholog with phenotype in [30] - see text for details

sequences formed distinct clades corresponding to the eight known $\mathrm{ABC}$ transporter subfamilies $\mathrm{A}$ to $\mathrm{H}$ (Fig. 2; Additional file 5: Figure S3).

\section{Gene expression across developmental stages}

Since prior research in T. castaneum revealed that only 10 $\mathrm{ABC}$ transporters had obvious phenotypic consequences following RNAi-mediated knockdown [30], our study focused on functional analysis of their predicted $D . v$ virgifera orthologs. From this list, our initial predictions from the D. v. virgifera transcriptome (DNASTAR assembly) identified eight orthologs (Table 3). Differences resided in that $T$. castaneum has two closely related $A B C A$ genes (TcABCA-9A and TcABCA-9B) which appear to represent a $T$. castaneum-specific duplication (Additional file 5: Figure S3). We were unable to identify a direct ortholog for these genes, but the closest homolog we found in the $D . v$. virgifera transcriptome appeared to be DvvABCA_50718. Analogously, we were unable to identify a direct ortholog to TcABCG-8A, so we targeted DvvABCG_14042, the closest identifiable homolog according to BLASTp results. Finally, while the ABCG genes TcABCG-9A and TcABCG$9 B$ represent the orthologs of the $T$. castaneum eye-color genes scarlet and white, respectively [32], results of BLASTx searches of the DNASTAR assembly resulted only in the identification of an ortholog of white, $D v v w$ [46]. Semi-quantitative PCR of these eight D. v. virgifera $\mathrm{ABC}$ transcripts showed that all are expressed across all of the developmental stages examined (Fig. 3a).

\section{RNAi knockdown phenotypes}

Different growth stages of $D$. v. virgifera were microinjected with dsRNAs (Table 3), after which the level of each corresponding transcript was below or nearly below semiquantitative PCR detection limits. Specifically, the level of each targeted $D$. v. virgifera transcript was reduced at 5days post-injection as compared to buffer-injected controls (Fig. 3b). Moreover, injection of each of the eight dsRNAs resulted in defined phenotypes among dsRNA treated cohorts (Table 3; Fig. 4). The knockdown of DvvABCA_50718 led to approximately $60 \%$ mortality among treated prepupae, compared to $5 \%$ for the buffer-treated control group. In addition, the adults that survived pre-pupal injection and successfully eclosed had defects in their wings and elytra (Fig. 4a), while no phenotypic effects were observed among buffer-injected controls. Injection of $D v v A B C B_{-}$ 


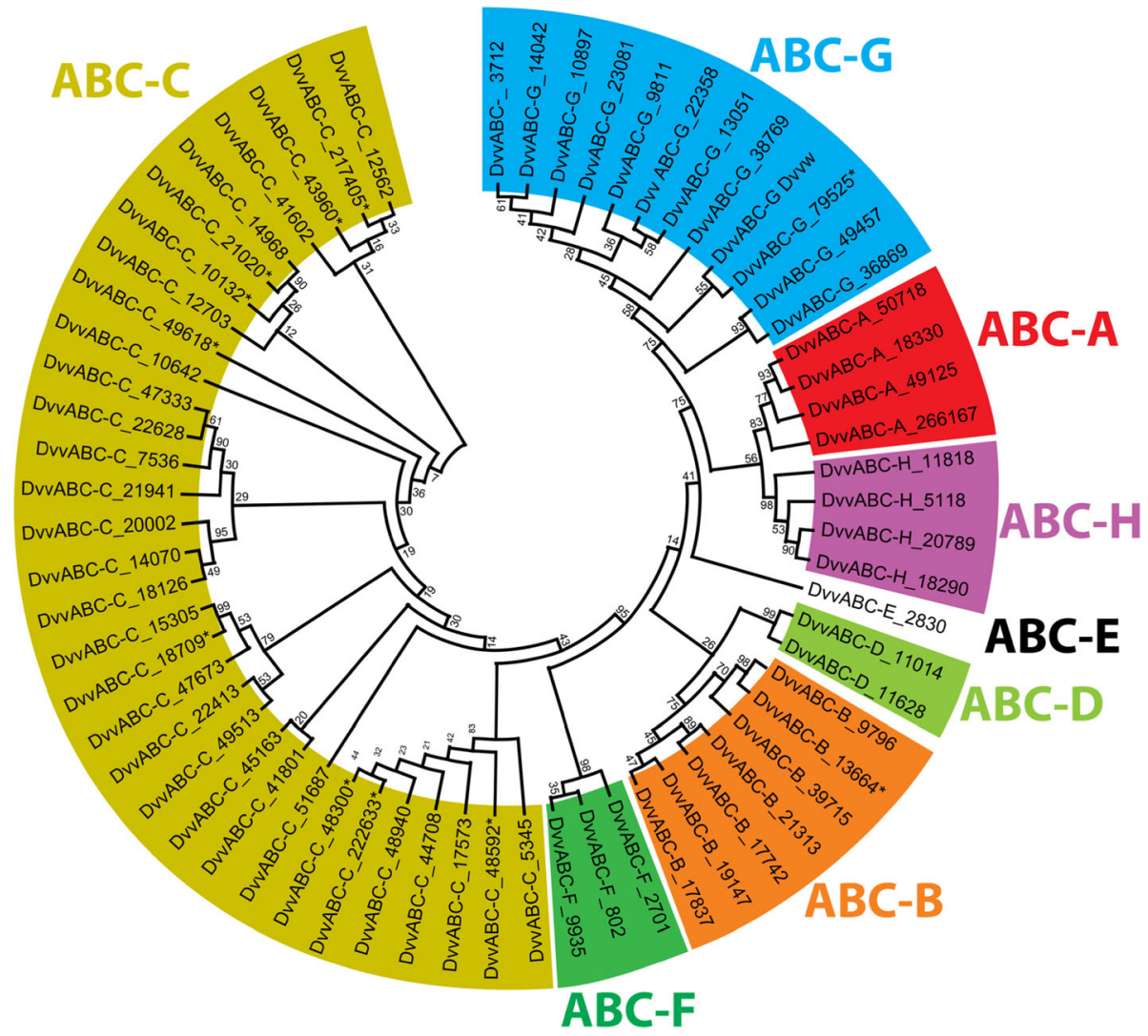

Fig. 2 Intraspecific phylogenetic relationships among D. v. virgifera $A B C$ transporters. Clades corresponding to subfamilies $A-H$ are indicated by color. Bootstrap values are given at the internodes as percentage of 1000 pseudoreplicates

39715 dsRNA into larvae resulted in 100\% mortality, and injections into pre-pupae led to defects in their development, which caused individuals to be unable to complete the pupal-adult molt and ultimately resulted in 100\% mortality (Fig. 4b). Knockdown of $D v v A B C B \_39715$ in newly-eclosed adult female $D$. v. virgifera resulted in significant reduction in egg laying compared to untreated females (Fig. 5a). Upon further investigation, we discovered that injection of this dsRNA also affected ovary development, causing underdeveloped ovaries, hence the failure to produce eggs (Table 3; Fig. 4j).

Table 3 Results of RNAi knockdown of selected ABC transporters

\begin{tabular}{|c|c|c|c|c|}
\hline Transcript & Stage & $\mathrm{KD}$ & Phenotype & Figure \\
\hline DvvABCA_50718 & Pre-pupal & $60 \%$ & Deformed wings \& elytra & $4 \mathrm{~A}$ \\
\hline \multirow[t]{3}{*}{ DvvABCB_39715 } & Larval & $100 \%$ & Lethal & NS \\
\hline & Pre-pupal & $100 \%$ & Defect in pupal-adult molt & $4 \mathrm{~B}$ \\
\hline & Eclosed females & $0 \%$ & Malformed ovaries; low egg lay & 4J \\
\hline DvvABCE_2830 & Larval & $100 \%$ & Lethal & $4 \mathrm{G}$ \\
\hline DvvABCF_2701 & Larval & $100 \%$ & Lethal & $4 \mathrm{H}$ \\
\hline \multirow[t]{2}{*}{ DvvABCG_3712 } & Pre-pupal & $80 \%$ & Lethal; pupal developmental arrest & $4 C$ \\
\hline & Eclosed females & $0 \%$ & Prevented embryonic development & 41 \\
\hline Drvw & Pre-pupal & $0 \%$ & Pigmentation defect; white eyes & $4 \mathrm{E}$ \\
\hline \multirow[t]{2}{*}{ DvvABCG_14042 } & Larval & $100 \%$ & Lethal at molting & NS \\
\hline & Pre-pupal & $80 \%$ & Lethal pupal developmental arrest & $4 \mathrm{D}$ \\
\hline DvvABCH_5118 & Larval & $100 \%$ & Lethal at molting & NS \\
\hline
\end{tabular}




\section{A) Stage Specific}

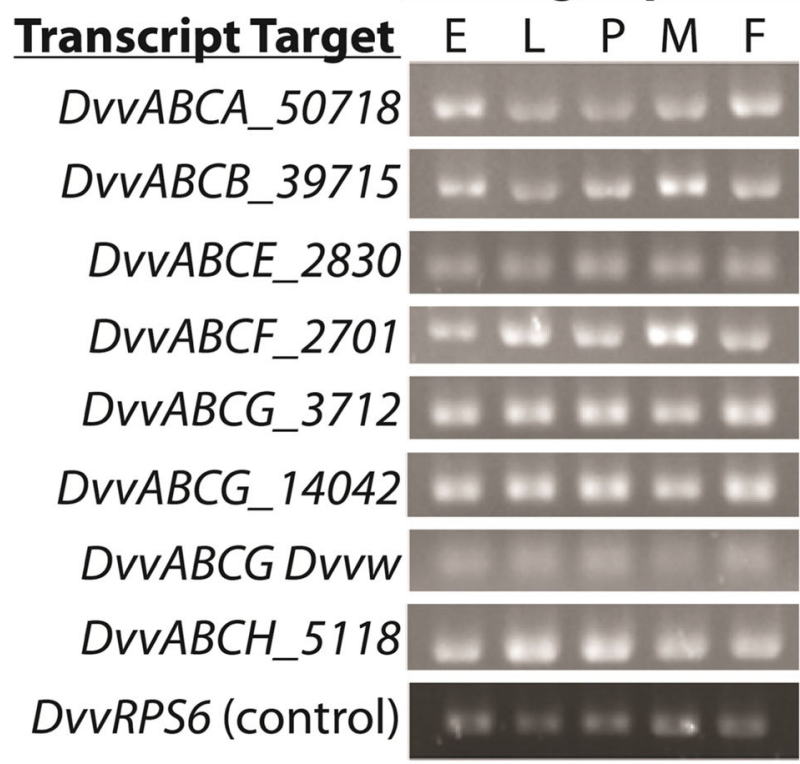

B) Injected

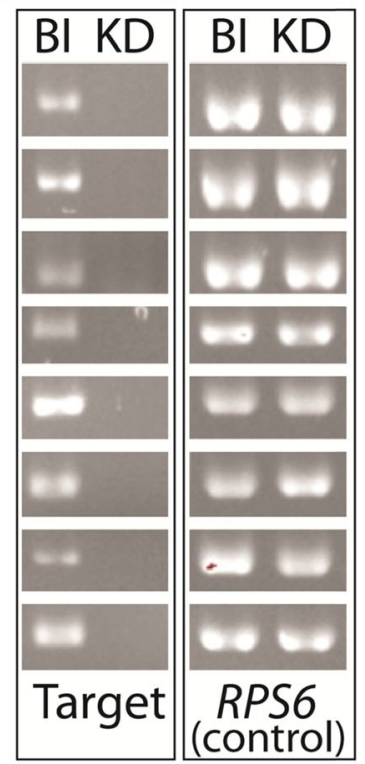

Fig. 3 Semi-quantitative PCR results of select D. v. virgifera $A B C$ genes. a Developmental stage-specific expression profile of select transcripts. RNA isolated from Eggs (E), Larvae (L), Pupae (P), and adult Males (M) and Females (F) for each of the eight genes. DvvRPS6 was used as a positive control. b Assessment of target RNA levels in injected individuals. RNA was isolated 5 days after injection from pools of buffer injected (BI) individuals, and of dsRNA injected (KD) individuals. DvvRPS6 was used as a control to assess template quality

RNAi-mediated knockdown of DvvABCE_2830 and DvvABCF_2701 in larvae resulted in $100 \%$ mortality. Prior to death, it was noted that the body mass of treated individuals was less than that of similarly-aged larvae treated with buffer alone (Fig. 4g, h). Analogously, injection of $D v v A B C E \_2830$ and $D v v A B C F \_2701$ dsRNA separately into pre-pupae both caused $100 \%$ mortality with no adult eclosion (results not shown). Injection of dsRNA specific for $D v v A B C H \_5118$ into early-instar $D$. v. virgifera larvae and pre-pupae caused development to arrest as individuals prepared to molt, thus resulting in $100 \%$ mortality (Fig. 4f). Affected individuals appeared to desiccate prior to death (personal observation).

Injection of dsRNA targeting DvvABCG_3712, DvvABCG_14042, and Dvvw resulted in phenotypes similar to those seen with RNAi knockdown of the corresponding T. castaneum orthologs [30]. Specifically, injection of dsRNA targeting Dvvw, gave the expected white-eye phenotype (Fig. 4e); indeed, we had identified this white ortholog previously [46]. Injection of DvvABCG_3712 dsRNA into pre-pupae caused developmental defects that resulted in $80 \%$ mortality (Table 3; Fig. 4c). Interestingly, adult females treated with DvvABCG_3712 dsRNA produced fewer eggs compared to females injected with buffer alone (Fig. 5b), and the eggs that were laid lacked obvious signs of embryonic development (Fig. 4i) and ultimately failed to hatch (Additional file 6: Figure S4). Injection of DvvABCG_14042 dsRNA into larvae and pre-pupae resulted in molting defects; about $80 \%$ of these died during their next molt (Table 3), while the $20 \%$ that survived through subsequent larval molts died following pupation (Fig. 4d).

\section{Discussion}

In recent years, $\mathrm{ABC}$ transporters have become a major focus for research in arthropods. This is in part due to their overall role in xenobiotic transport and insecticide resistance $[25,47-50]$, but more specifically, due to their suspected role in susceptibility to Bt toxins [38, 51, 52]. For example, Gahan et al. [53] reported genetic linkage of Heliothis virescens $H v A B C C 2$ with resistance to Cry1Ac, while changes in the structure, splicing, or expression level of $A B C C 2$ orthologs were later associated with Cry1Ac resistance in Helicoverpa armigera [54], Bombyx mori [55], and Spodoptera exigua [56]. Indeed, expression of the P. xylostella ABCC2 ortholog in Drosophila melanogaster conferred susceptibility to this lepidopteran-specific toxin [57]. An $A B C C 2$ ortholog is also linked to Cry1F resistance in Ostrinia nubilalis [58] and S. frugiperda [59]. Additionally, structural mutations in a member of subfamily $\mathrm{A}, H a A B C A 2$, were implicated in Cry2Ab resistance in $H$. armigera [60], and, more recently, researchers were able to recapitulate an $A B C A 2$ resistance allele in a susceptible population of $H$. armigera [61], providing further evidence for the importance of normal $A B C A 2$ function in Cry2Ab toxicity. Reduced expression of ABCG members have been associated with 

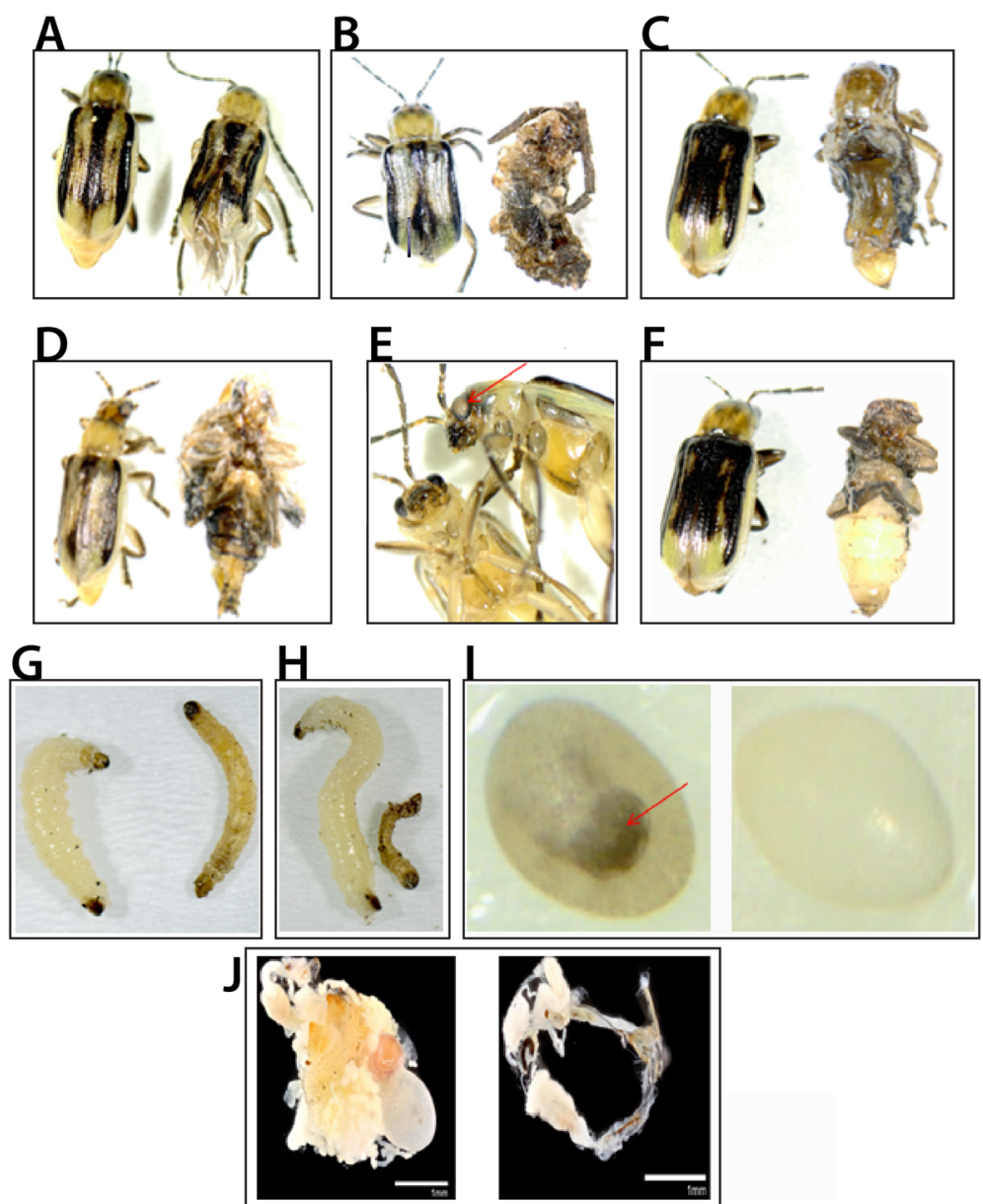

Fig. 4 Effects of DVVABC transporter-specific RNAi on D. v. virgifera development. Buffer-injected controls (right) are shown next to dsRNA-injected individuals. a Injection of DvrABCA_50718-specific dsRNA into pre-pupae (PP) caused defects in adult wing development, while b PP injection of dsRNA for DvvABCB_39715 caused molting defects during eclosion. $\mathbf{c}, \mathbf{d}$ and $\mathbf{f}$ Injection of dsRNAs for DvvABCG_3712, DvvABCG_14042 or DvvABCH_5118 into PP each resulted in severe molting defects during eclosion. e PP injection of Dvrw-specific dsRNA caused loss of eye pigmentation (arrow), while ( $\mathbf{g}-\mathbf{h})$ larval injection of DVVABCE_2830 or DVVABCF_2701 resulted in a reduction in body mass and death prior to molting. i Injection of DVVABCG_3712-specific dsRNA into adult females interfered with embryonic development (arrow indicates location of head capsule in a control embryo). j DvVABCB_39715-specific dsRNA injected into adult females disrupted ovary development

Cry1Ac resistance in P. xylostella [62], as well as Cry1Ac and Cry1 $\mathrm{Ab}$ resistance in O. furnacalis [63]. More recent studies in species of Coleoptera have implicated $\mathrm{ABCB}$ subfamily members in Cry3Aa resistance in C. tremula [38] and in Cry3Ab1 resistance in D. v. virgifera [39].

The study of $\mathrm{ABC}$ transporters in several arthropod species have relied on genomic data, including $T$. castaneum [30], Aethina tumida [64], B. mori [33], D. melanogaster [28], Bemisia tabaci [50], Daphnia pulex [65], and Tetranychus urticae [66]. Due to the status of D. v. virgifera as a major pest of cultivated maize (see Introduction) and current fragmented state of the unpublished draft genome assembly of this species (GenBank accession PXMJ00000000.2), the Illumina-based transcriptome assemblies reported here represent a particularly valuable genetic tool for gene discovery, characterization, and genome annotation. In particular, the $65 \mathrm{ABC}$ transporter genes we identified are expected to be useful in downstream studies on insecticide resistance traits in D. v. virgifera.

Broehan et al. [30] previously identified $73 \mathrm{ABC}$ transporters in $T$. castaneum, and a 74th $\mathrm{ABC}$ transporter was more recently reported by Grubbs et al. [32]. There are sev-

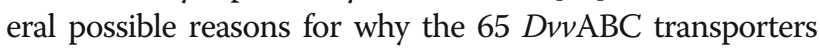
we identified are comparatively fewer than in T. castaneum. Firstly, our transcriptome was derived from lowerthroughput sequencing data (Illumina MiSeq), therefore genes expressed at very low levels may not have been represented within our raw Illumina data. Secondly, our RNAseq libraries were not comprehensive of all possible life/ growth stages or conditions, such that transcripts not expressed during growth states or under conditions used in 


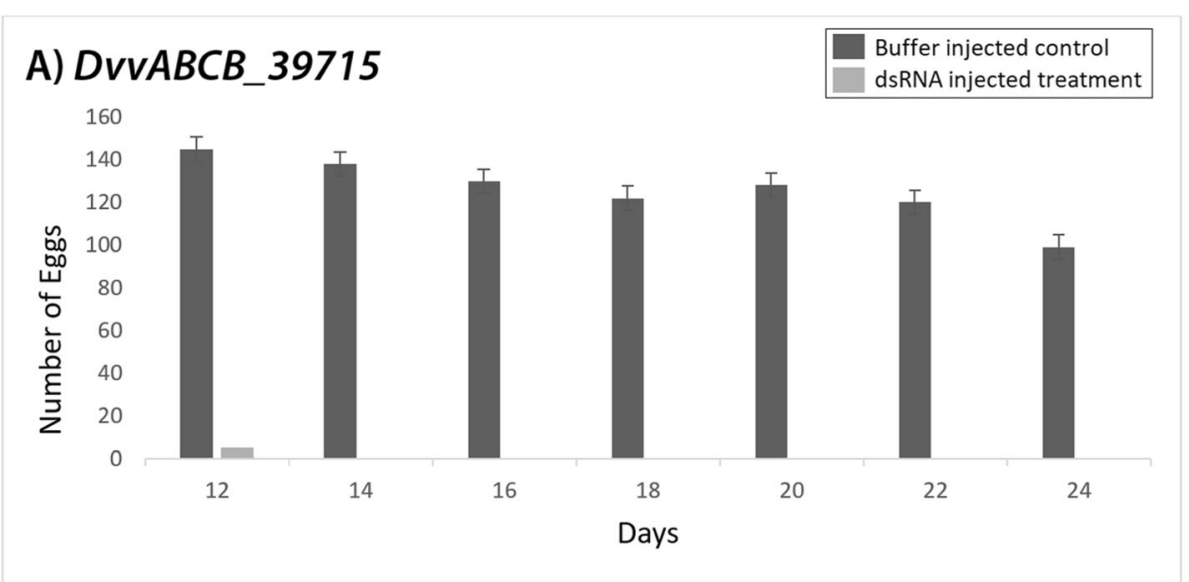

\section{B) DvvABCG_3712}

Buffer injected control dsRNA injected treatment

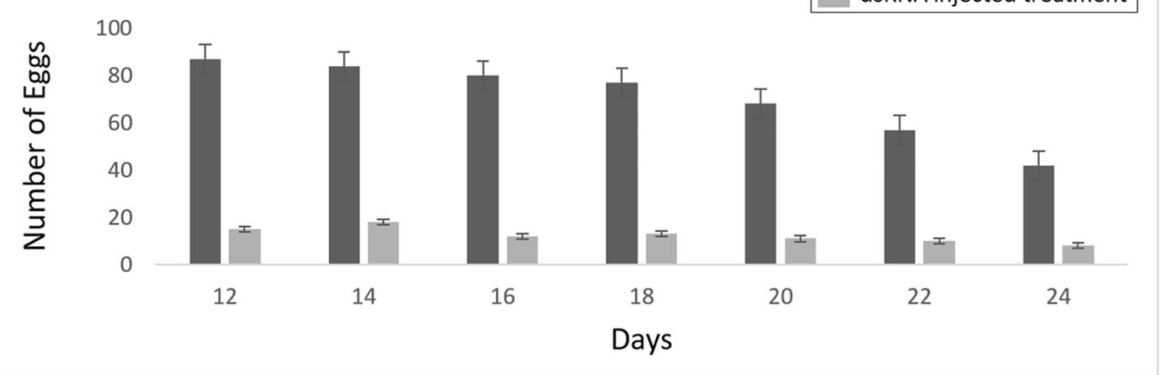

Fig. 5 Effects of injected dsRNA on egg laying. Effects on oviposition following injections of dsRNA targeting a DvVABCB_39715 and $\mathbf{b}$ DvvABCG_3712. Females injected with DvvABCB_39715 dsRNA failed to lay eggs, and those injected with DvvABCG_3712 dsRNA laid fewer eggs and those that were laid failed to develop. In both cases, buffer-injected females lay near-normal numbers. The eggs laid within a period of 2 weeks were counted every other day

this study would have been missed. Regardless, BLASTx analyses of the 65 putative $D$. v. virgifera $\mathrm{ABC}$ transporters identified in this study demonstrate their greatest sequence similarity to $T$. castaneum and A. glabripennis orthologs. This is probably a consequence of the extensive publicly available genomic data for both $T$. castaneum and A. glabripennis, as well as their close phylogenetic relationships to $D$. v. virgifera. Furthermore, the putative one-to-one relationship among orthologs from $D$. v. virgifera and T. castaneum may suggest the retention of copy number without extensive gene loss or gain across evolutionary time.

Despite the relatively large amount of genomic and transcriptomic data available for model and some nonmodel coleopteran species, there is a comparative overall dearth of functional data available to support automated computational annotations. To partially address this shortfall, we generated functional information based on RNAi knockdown of eight $D$. v. virgifera $A B C$ transporters, each of which demonstrated fairly conserved roles relative to their T. castaneum orthologs [30]. While some $D . \quad v$. virgifera RNAi-mediated loss-of-function phenotypes include visible developmental defects, such as loss of eye pigmentation, others cause growth arrest and/or death. For example, knockdown of $D v v A B C A_{-}$ 50718 led to death during the pupal-to-adult molt and also caused deformation of wings and elytra in surviving adult beetles, which was the same as previously seen in T. castaneum [30] (Table 3; Fig. 4a). Since subfamily A transporter members are implicated in mammals with lipid transport, which can impact cell physiology [67], it is conceivable that the effects of the knockdown of DvvABCA_50718, and of its homologs, TcABCA-9A/9B, in $T$. castaneum [30], could be the result of disrupting critical lipid transport. $D v v A B C B \_39715$ RNAi also recapitulated the lethal effects of its $T$. castaneum ortholog; the effects on female fecundity could make this gene a particularly interesting target for RNAi-based pest control. It is worth noting that $D$. v. virgifera is predicted to have one more $\mathrm{ABCB}$ HT subfamily member compared to other insects [25], especially other beetles [30, 64]. While ABCB FTs have been implicated in chemical insecticide resistance among insects [47], HTs are known to be mitochondrial transporters in humans, with roles in iron metabolism and transportation of $\mathrm{Fe} / \mathrm{S}$ protein precursors $[68,69]$. These possibilities were outside the scope of our research, but future investigations into the 
function of $D v v \mathrm{ABCBs}$ could be beneficial for deciphering mechanisms of resistance evolution in $D$. v. virgifera.

RNAi knockdown of DvvABCE_2830 and DvvABCF 2701 resulted in $100 \%$ larval mortality. $\mathrm{ABCE}$ and $\mathrm{ABCF}$ subfamilies are highly conserved across all phyla, and due to their lack of TMDs are considered non-transporters. Instead, they appear to play roles in regulating translation $[70,71]$, indicating that $\mathrm{ABCE}$ and $\mathrm{ABCF}$ proteins are essential. Thus, given that these genes are highly conserved across taxa in sequence, function, and RNAi phenotype [30], it may not be surprising that lethal RNAi knockdown phenotypes were obtained in $D$. v. virgifera.

The phenotypes observed following independent RNAi knockdown of DvvABCG_14042 and DvvABCH_5118 involved molting defects that resulted in near complete mortality. While these results are consistent with functional analysis of their $T$. castaneum orthologs, RNAi knockdown of the DvvABCG_14042 homolog TcABCG$8 A$ in $T$. castaneum produced an additional phenotype of premature development of compound eyes [30]. In contrast, we did not observe any analogous eye phenotypes in $D . v$. virgifera following RNAi knockdown. It is likely that since the injected $D . v$ virgifera larvae died prior to reaching the next stage of development, there was no opportunity for compound eyes to form. In other species, orthologs of $D v v A B C H \_5118$ are known to transport cuticular lipids that are deposited in the outer epicuticle layer to form a waterproof barrier [30, 62]. Therefore, it could be that cuticular lipid deposition may be reduced following RNAi knockdown of this $\mathrm{ABCH}$ transporter, which could promote desiccation and subsequent mortality of affected individuals.

The ABCG proteins are HTs, and, with 12 predicted members, form the second largest subfamily of $A B C$ transporters identified in D. v. virgifera (Table 2). Among insects, some of the first ABCGs to be characterized were the pigment transporters (white, scarlet and brown) in $D$. melanogaster [34, 72]. Mutants of white are characterized by white eyes (i.e. complete loss of eye pigmentation), scarlet mutants by bright red eyes (i.e. loss of brown pigments), and brown mutants by dark brown eyes (i.e. loss of red pigments) [34]. Studies have revealed that some ABCG proteins perform other crucial physiological roles in the transport of lipids, sterols, and drugs [73]. In the current study, RNAi-mediated knockdown of Dvvw resulted in a white-eyed phenotype consistent with prior observations in T. castaneum [30], and with our own previous findings in $D . v$. virgifera [46]. Our findings support a prediction that $D v v w$ is part of the ommochrome pathway, where it is likely acting within a heterodimeric complex to import ommochrome pigments into the pigment granules of the compound eye. As mentioned above, loss of white function in D. melanogaster, results in white-eyed flies, while mutations in scarlet lead to red-eyed flies.
However, RNAi-mediated knockdown of the corresponding gene, ABCG-9A (scarlet), in T. castaneum produces white-eyed beetles [30, 32]. This finding was not surprising, since a previous report of RNAi targeting vermilion, a pivotal gene in the ommochrome pathway, also generates a white-eyed phenotype in T. castaneum [74], leading the authors to conclude that the $T$. castaneum eye is pigmented by ommochromes alone, and that the ommochrome biosynthetic pathway in T. castaneum produces red pigments as end products, rather than brown pigments as in D. melanogaster. Unfortunately, our initial survey of the D. v. virgifera transcriptome failed to identify a scarlet ortholog in our DNASTAR assembly, thus its function was not assessed. We did identify a scarlet ortholog from the Trinity assembly (See Table 2 and Additional file 5: Figure S3) after we had completed our functional analyses, but we were still unable to find any evidence of a brown ortholog. So, it will be interesting to investigate in future studies if pigmentation of the $D$. v. virgifera eye is more similar to that of T. castaneum or D. melanogaster. Specifically, in D. melanogaster a third ABCG transporter, brown, is required for wild-type pigmentation of the eye. In flies, Brown heterodimerizes with White and transports pteridine-based pigments into the eye. Although an ortho$\log$ of brown has been identified in the T. castaneum genome, no function has been identified [32].

\section{Conclusion}

This study provides a relatively large transcriptomic resource comprising genes expressed across several life stages of the arthropod pest species, $D$. v. virgifera. Due to potential omission of orthologs from our assembly, undoubtedly additional research will need to be performed in order to identify the full compliment of $A B C$ transporters encoded by $D$. v. virgifera, and further functional assays will be needed to validate putative biochemical roles. Regardless, our work represents the initial description of the $A B C$ transporter gene family in $D$. v. virgifera. Furthermore, the knockdown of $A B C$ transporters DvvABCB_39715 and DvvABCG_3712, each of which reduced egg production and/or prevented embryonic development, could provide novel targets for $D$. v. virgifera population suppression and use as an insecticidal control agent. This research is a contribution to a growing set of genomic resources for arthropods, and provides information that may facilitate the development of methods to enhance the control of a devastating agricultural pest species.

\section{Methods}

\section{Insect rearing}

All D. v. virgifera used in this study are nondiapausing, from a colony previously established at North Carolina State University using beetles obtained from both Dr. 
Wade French (USDA-ARS-NGIRL, Brookings, SD) and Crop Characteristics, Inc. (Farmington, MN, USA) (see [75]). Eggs deposited in an oviposition chamber (agar plate with cheese cloth) were collected weekly, pipetted into soil-filled containers, and held at $26^{\circ} \mathrm{C}$ for 1 week. Larvae were reared on roots of germinated corn seed in $16-\mathrm{oz}$ containers, while adults were maintained in a $30 \mathrm{~cm}^{3}$ BugDorm (MegaView Science, Taiwan) at $26^{\circ} \mathrm{C}$, 70\% relative humidity with an L14:D10 photoperiod and fed an artificial diet (Western Corn Rootworm w/o Pollen Substitute, Frontier Insect Diet, Newark, DE, USA). Injected individuals were reared in small containers with corn seedlings to allow downstream observation.

\section{Transcriptome sequencing, assembly, and annotation}

Total RNA was extracted from mixed-staged $D$. v. virgifera embryos $(n=500$ from an overnight egg lay aged up to 14 days), mixed-stage larvae (first-instar larvae $(n=$ $20)$; second-instar larvae $(n=10)$; and third-instar larvae $(n=2)$, as well as an adult male, and an adult female ( $n=1$ each) using the RNeasy Mini Kit (Qiagen, Hilden, Germany) and treated with DNase I (Qiagen) according to the manufacturer's instructions. The isolated total RNA was submitted to the Genomic Sciences Laboratory (North Carolina State University, NC, USA) for quality assessment, poly(A) selection, fragmentation, selection of $\sim 650$ bp fragment sizes, Illumina TruSeq ${ }^{\circ}$ library preparation, and $300 \mathrm{bp}$ paired-end sequencing on an Illumina MiSeq sequencer (Illumina, San Diego, CA, USA).

Raw FASTQ reads for each library were assessed using FastQC [76]. Reads were initially imported into SeqMan NGen - (DNASTAR, Madison, WI, USA), where onboard scripts were used to quality trim and de novo assemble reads into contigs using default settings. Additionally, raw reads from individual libraries were trimmed of Illumina adapter sequence contamination, bases having Phred quality score $<20(q<20)$, and sequence reads $<35 \mathrm{bp}$ using Trimmomatic 0.32 [77]. Resulting trimmed read pairs from each library were concatenated into single R1- and R2-specific FASTQ files using a custom PERL script, and then assembled into contigs using SOAPdenovo-Trans v 1.0.3 [78] (asmflags $=0$; max_rd_len $=301$; map_len $=75$; avg_ins $=700$; kmer $(-K=127))$. Trimmed reads were also assembled with Trinity [79] using default parameters, except for adjustment for library insert length (--group_pairs_distance $=700$ ) and minimum read overlap (--path_ reinforcement_distance $=75$ ). The complexity of SOAPdenovo-Trans and Trinity assemblies were reduced by clustering allelic variants using CD-HIT-EST [80] with default parameters, except for change of sequence identity ( $-\mathrm{c} 0.95)$, word length $(-\mathrm{n} 10)$, and length of throw-away sequence $(-111)$. The relative completeness of each clustered $D . v$. virgifera transcriptome assembly was evaluated by comparison with the universal single-copy orthologs from Arthropoda obtained from OrthoDB v 9 [81] using BUSCO v 3 [82] ( $E$ value cutoff 0.001$)$. Full- and partial-length open reading frames and corresponding derived amino-acid sequences were predicted from the resulting SOAPdenovo-Trans clusters with TransDecoder v3.0.0 [83] using a minimum length of 100 amino acids.

The transcript sequences assembled by SeqMan NGen $^{\circ}$ (DNASTAR, Madison, WI) were imported into Blast2GO v4.0 [84, 85] and annotations acquired via BLASTx [86] comparison to the non-redundant (nr) arthropod-specific protein database at the National Center of Biotechnology Information (NCBI). The combined graphs were created at level 2 for Biological Process (P), Cellular Component (C), and Molecular Function (F) categories from Blast2GO.

\section{Bioinformatic analysis of the $D$. v. virgifera $A B C$ transporter family}

A searchable database was created from the combined DNASTAR D. v. virgifera transcript assembly, and subsequently searched with the set of deduced $T$. castaneum $\mathrm{ABC}$ transporter amino-acid sequences [30,32] as queries using the tBLASTn algorithm in BlastStation software (TM Software Inc., Arcadia, CA, USA). Homologous sequences were selected based on sequence identity and $E$ value $\left(<10^{-6}\right)$. Putative D. v. virgifera $\mathrm{ABC}$ sequences were then used as BLASTx queries of the non-redundant NCBI protein database using the web blast interface (https://blast.ncbi.nlm.nih.gov/Blast.cgi) to confirm their identity as insect $\mathrm{ABC}$ genes; those that appeared to not be of non-insect origin, or were otherwise not $A B C$ genes, were discarded. The number and positions of transmembrane domains were assessed via query of the NCBI Conserved Domain Database [87]. Finally, each D. v. virgifera $\mathrm{ABC}$ gene was putatively assigned to a subfamily (A-H) based on greatest similarity assigned to orthologs within BLASTx results. This BLAST search procedure was analogously repeated for SOAPdenovo-Trans and Trinity assemblies. The complexity of each $\mathrm{ABC}$ gene set was reduced by clustering allelic variants (sequence) across assemblies, and a comprehensive non-redundant set of putative $D$. v. virgifera $\mathrm{ABC}$ transporter contigs were generated (Additional file 7). Assembly of origin is denoted in sequence names as follows: DNASTAR (D), Trinity $(\mathrm{T})$, and SOAPdenovo-Trans $(\mathrm{S}=$ "scaffold" and $\mathrm{C}=$ "contig") within the FASTA files. The full translation product of each contig can be found in Additional file 8.

Phylogenetic relationships among derived $D$. v. virgifera $\mathrm{ABC}$ transporter protein sequences were reconstructed from the conserved NBD. A multiple sequence alignment was performed with MUSCLE using MEGAX 
[88] (default parameters) and used within a subsequent phylogenetic analysis. The unrooted Maximum Likelihood phylogenetic trees were constructed in the MEGAX program using default parameters in all categories except: LG model of amino-acid substitution with Gamma distributed substitution rates (based on Best Model determination within the MEGA program), Partial Deletion treatment of gaps/missing data, and 1000 bootstrap replicates [89]. ABC transporter subfamilies were assigned to $D$. v. virgifera sequences and clades within this phylogenetic analysis by comparison to similarities from our BLASTx search results and tree topologies among nearest orthologous gene family members in T. castaneum [30,32], and D. melanogaster. Multiple sequence alignments were generated as described above, wherein the deduced $D$. v. virgifera amino-acid sequences included full-length sequences when possible, but some were incomplete partial-protein sequences. All phylogenetic reconstruction methods were performed as described above.

\section{Gene expression across developmental stages}

Preliminary analysis to estimate the relative expression levels for eight transcripts (DvvABCA_50718, DvvABCB 39715, DvvABCE_2830, DvvABCF_2701, DvvABCG_ 3712, DvvABCG_14042, Dvvw and DvvABCH_5118) across growth stages was made via semi-quantitative PCR in order to ensure dsRNA injections would be performed prior to the time of corresponding peak expression. Total RNA was extracted from each developmental stage [embryo (E), larval (L), pupal (P), and adult male (M) and female (F)], from which cDNA was reverse transcribed using the Superscript ${ }^{\mathrm{Tm}}$ III First-Strand Synthesis System (Invitrogen, Carlsbad, CA, USA) using an anchored poly $(\mathrm{T})$ primer. These cDNA pools were then used individually as template in eight separate PCR reactions each using $D$. v. virgifera $\mathrm{ABC}$ transporter transcript-specific primer pairs (Additional file 9: Table S3). Primers for the D. v. virgifera ribosomal protein S6, DvvRPS6, were used as an external control. PCR reactions were set up using MyTaq ${ }^{\text {TM }}$ DNA polymerase according to manufacturer instructions (Bioline, Memphis, $\mathrm{TN}$, USA), and subsequent amplification reactions were performed in a C1000 Thermal Cycler (Bio-Rad Laboratories Inc., Hercules, CA, USA) with the following cycling conditions: $\left(95^{\circ} \mathrm{C}\right.$ for $\left.3 \mathrm{~min}\right), 25 \times\left(95^{\circ} \mathrm{C}\right.$ for 30 s, $58^{\circ} \mathrm{C}$ for $30 \mathrm{~s}, 72{ }^{\circ} \mathrm{C}$ for $\left.10 \mathrm{~s}\right)$, (4 min incubation at $72{ }^{\circ} \mathrm{C}$ ). Amplification products were then visualized and compared using $1.5 \%$ agarose gel electrophoresis.

\section{RNAi knockdown phenotypes}

Primers were designed for the generation of dsRNA using Vector NTI Advance (VNTI) software (Invitrogen), for all $\mathrm{ABC}$ genes whose orthologs are known to produce obvious RNAi phenotypes in T. castaneum [30]. These primer sets targeted regions that encoded transcriptspecific TMD domains; this was done in order to potentially reduce unintended off-target effects by avoiding the more conserved NBD domains. Partial cDNAs were amplified for the 8 genes (DvvABCA_50718, DvvABCB_39715, $D v v A B C E \_2830, \quad D v v A B C F \_2701, \quad D v v A B C G \_3712$, $D v v A B C G \_14042$, Dvvw and $\left.D v v A B C H \_5118\right)$, as described above for developmental stage expression. Nested PCR was performed with an initial denaturation of $95^{\circ} \mathrm{C}$ for $3 \mathrm{~min}, 35$ cycles at $95^{\circ} \mathrm{C}$ for $30 \mathrm{~s}, 58^{\circ} \mathrm{C}$ for $30 \mathrm{~s}$, and $72{ }^{\circ} \mathrm{C}$ for 10 s, and then a 4 min incubation at $72^{\circ} \mathrm{C}$ on a C1000 Thermal Cycler (Bio-Rad). PCR products were purified using the QIAquick PCR Purification Kit (Qiagen) according to the manufacturer's instructions, ligated into the pGEM-T vector (Promega, Madison, WI, USA), and the resulting plasmids were used to transform TOP10 competent E. coli (Invitrogen). All positive clones were cultured in a selective LB medium containing $100 \mathrm{mg}$ ampicillin $\mathrm{L}^{-1}$. The recombinant plasmid DNAs were isolated using the QIAprep ${ }^{\circ}$ Spin Miniprep Kit (Qiagen), and the inserts were Sanger sequenced and confirmed by use as BLASTn queries (https://blast.ncbi.nlm.nih.gov/Blast. cgi). Purified plasmids with each cloned $\mathrm{ABC}$ transporter were used as template in separate PCR reactions primed with the following primers: T7 as a forward primer (due to location in pGEM), and a pGEM-specific reverse primer that was tailed with T7. This enabled all amplification reactions to be performed using the same set of primers under conditions described above. PCR products were analyzed by $1.5 \%$ agarose gel electrophoresis, purified using the QIAquick PCR Purification Kit (Qiagen), and then $1 \mu \mathrm{g}$ of each was used as template for dsRNA synthesis using the MEGAscript T7 in vitro Transcription Kit (Ambion, Austin, TX, USA). Each of the synthesized dsRNAs were purified using the MEGAclear Kit (Ambion) and concentration determined using a Nanodrop 1000 (Thermo Scientific, Waltham, MA, USA) using the singlestranded RNA setting.

RNAi assays were conducted by injecting dsRNA corresponding to each of the 8 specific $D$. v. virgifera $A B C$ genes individually into the hemocoel of third-instar larvae, pre-pupae and/or newly-eclosed female adults. Before microinjection, experimental insects were anesthetized on ice for $30 \mathrm{~min}$, then injected with $\sim 0.2 \mu \mathrm{l}$ of a genespecific dsRNA at a concentration of 1-2 $\mu \mathrm{g} / \mu \mathrm{l}$. Each treatment was replicated three times, with $\geq 20$ individuals in each replicate. Following injection, larvae and pre-pupae were allowed to recover at room temperature for 1 hour, and then moved to germinated corn for further monitoring and phenotypic analysis. Phenotypes were observed daily using a stereomicroscope, and transcript levels assessed at 5 days post-injection by semi-quantitative PCR using RNA isolated from pools of injected individuals 
(one individual per replicate, for a total of three individuals per PCR reaction).

Treated females were kept in an oviposition chamber (agar plate with cheese cloth) and maintained on an artificial diet. At 2 days post-injection, females were mated to untreated males, and generally started to lay eggs 10 days later. To determine egg viability, eggs were harvested from the oviposition chamber and placed on moistened filter paper in Petri dishes and held at $26^{\circ} \mathrm{C}$, $70 \%$ relative humidity with an L14:D10 photoperiod. Females were allowed to lay eggs over a two-week period, and eggs were counted every other day to assess the rate of egg laying. Hatch rate counts were made every other day, beginning 10 days after the first egg lay (22-days post-injection) and continuing for 4 weeks until no further hatching was observed.

\section{Supplementary information}

Supplementary information accompanies this paper at https://doi.org/10. 1186/s12864-019-6218-8

Additional file 1: Table S1. Comparisons among D. v. virgifera reference transcriptome assemblies based upon total assembly output, number of transcript clusters, predicted open reading frames (ORFs), and benchmarking of single-copy orthologs (BUSCOs; Arthropoda $\vee 9$ reference set).

Additional file 2: Figure S1. Blast2GO annotation results for the combined D. v. virgifera transcriptome.

Additional file 3: Figure S2. Top-Hits species distribution from Blast2GO.

Additional file 4: Table S2. D. v. virgifera $A B C$ naming chart.

Additional file 5: Figure S3. Multispecies $A B C$ Protein Phylogeny. Additional file 6: Figure S4. Effect of DvvABCG_3712 RNAi on egg hatching.

Additional file 7. Nucleotide Sequences of Dvv ATP binding cassette (ABC) transporters

Additional file 8: Amino Acid Sequences of D. v. virgifera $A B C$ transporters.

Additional file 9: Table S3. Primer sequences used for dsRNA synthesis and RT-PCR analysis.

\section{Abbreviations}

ABC: ATP-binding cassette; Bt: Bacillus thuringiensis; GO: Gene ontology; NBD: Nucleotide-binding domain; NCBI: National Center of Biotechnology Information; ORFs: Open reading frames; TMD: Transmembrane domain

\section{Acknowledgements}

We thank Pei-Shan Wu, Sofia Pinzi, Teresa O'Leary, Lauren Slayton and Wanose Getachew for their expert assistance in rearing WCR. This article reports the results of research only and any mention of products or services does not constitute an endorsement by USDA-ARS. USDA-ARS is an equal opportunity provider and employer.

\section{Authors' contributions}

FA and MDL conceived and designed the experiments; FA performed the experiments; FA, NG, BC, BW, MDL analyzed the data; and FA, NG, BC, MDL wrote the manuscript. All authors have read and approved the manuscript.

\section{Funding}

This work was supported by a grant from the Monsanto Corn Rootworm Knowledge Research Program, grant number AG/1005 (to ML), and start-up funds to ML from NC State University. Portions of this work by BC was supported by a joint United States Department of Agriculture (USDA), Agricultural Research Service (ARS) (CRIS Project 5030-22000-018-00D), ARS SCINet computational resources (https://www.ars.usda.gov/scinet/), and the lowa Agriculture and Home Economics Experiment Station, Ames, IA (Project 3543). USDA-ARS is an equal opportunity employer and provider. The funding bodies had no role in the design of the study, collection, analysis, or interpretation of data, or in writing the manuscript.

\section{Availability of data and materials}

The datasets generated and/or analyzed during the current study are included in this published article [and its supplementary information files] or are available at NCBI under BioProject PRJNA490283, with SRA database accessions SRX4669438 to SRX4669441 for the raw reads, and TSA database accessions GHNH00000000 and GHRK00000000 for the Trinity and DNASTAR assemblies, respectively.

Ethics approval and consent to participate

Not applicable.

\section{Consent for publication}

Not applicable.

\section{Competing interests}

The authors declare that they have no competing interests.

\section{Author details}

${ }^{1}$ Department of Entomology and Plant Pathology, North Carolina State University, Box 7613, 1566 Thomas Hall, Raleigh, NC 27695-7613, USA. USDA-ARS, Corn Insects \& Crop Genetics Research Unit, Ames, IA 50011, USA.

Received: 21 March 2019 Accepted: 22 October 2019

Published online: 27 November 2019

\section{References}

1. Dillen K, Mitchell PD, Van Looy T, Tollens E. The western corn rootworm, a new threat to European agriculture: opportunities for biotechnology? Pest Manag Sci. 2010;66(9):956-66.

2. Levine $\mathrm{E}$, Oloumisadeghi $\mathrm{H}$. Management of Diabroticite Rootworms in corn. Annu Rev Entomol. 1991;36:229-55.

3. Levine E, Spencer JL, Isard SA, Onstad DW, Gray ME. Adaptation of the western corn rootworm to crop rotation: evolution of a new strain in response to a management practice. Am Entomol. 2002:48(2):94-117.

4. Sappington TW, Siegfried BD, Guillemaud T. Coordinated Diabrotica genetics research: accelerating progress on an urgent insect pest problem. Am Entomol. 2006;52(2):90

5. Gray ME, Sappington TW, Miller NJ, Moeser J, Bohn MO. Adaptation and invasiveness of Western corn rootworm: intensifying research on a worsening Pest. Annu Rev Entomol. 2009;54:303-21.

6. Mabry TR, Spencer JL. Survival and oviposition of a western corn rootworm variant feeding on soybean. Entomol Exp Appl. 2003;109(2):113-21.

7. Shaw JT, Paullus JH, Luckmann WH. Corn-rootworm (Coleoptera Chrysomelidae) Oviposition in soybeans. J Econ Entomol. 1978;71(2):189-91.

8. Roselle R, Anderson L, Simpson R, Webb M. Annual report for 1959, cooperative extension work in entomology. Lincoln: University of Nebraska Extension; 1959.

9. Pereira $A E$, Wang $H C$, Zukoff $S N$, Meinke $L$, French BW, Siegfried BD. Evidence of Field-Evolved Resistance to Bifenthrin in Western Corn Rootworm (Diabrotica virgifera virgifera LeConte) Populations in Western Nebraska and Kansas. PLoS One. 2015;10(11):e0142299.

10. Metcalf $\mathrm{R}$. The ecology of insecticides and the chemical control of insects. In: Ecological theory and integrated pest management practice. New York: Wiley; 1986. p. 251-97.

11. Wang H, Coates BS, Chen H, Sappington TW, Guillemaud T, Siegfried BD Role of a gamma-aminobutryic acid (GABA) receptor mutation in the evolution and spread of Diabrotica virgifera virgifera resistance to cyclodiene insecticides. Insect Mol Biol. 2013;22(5):473-84.

12. Gassmann AJ, Petzold-Maxwell JL, Keweshan RS, Dunbar MW. Field-Evolved Resistance to Bt Maize by Western Corn Rootworm. PLoS One. 2011:6(7): e22629. 
13. Gassmann AJ, Petzold-Maxwell JL, Clifton EH, Dunbar MW, Hoffmann AM, Ingber DA, Keweshan RS. Field-evolved resistance by western corn rootworm to multiple Bacillus thuringiensis toxins in transgenic maize. Proc Natl Acad Sci U S A. 2014;111(14):5141-6.

14. Zukoff SN, Ostlie KR, Potter B, Meihls LN, Zukoff AL, French L, Ellersieck MR, French BW, Hibbard BE. Multiple assays indicate varying levels of cross resistance in Cry3Bb1-selected field populations of the Western corn rootworm to mCry3A, eCry3.1Ab, and Cry34/35Ab1. J Econ Entomol. 2016;109(3):1387-98

15. Gassmann AJ, Shrestha RB, Jakka SRK, Dunbar MW, Clifton EH, Paolino AR, Ingber DA, French BW, Masloski KE, Dounda JW, et al. Evidence of resistance to Cry34/35Ab1 corn by Western corn rootworm (Coleoptera: Chrysomelidae): root injury in the field and larval survival in plant-based bioassays. J Econ Entomol. 2016;109(4):1872-80.

16. Ludwick DC, Meihls LN, Ostlie KR, Potter BD, French L, Hibbard BE. Minnesota field population of western corn rootworm (Coleoptera: Chrysomelidae) shows incomplete resistance to Cry34Ab1/Cry35Ab1 and Cry3Bb1. J Appl Entomol. 2017;141(1-2):28-40.

17. Wangila DS, Gassmann AJ, Petzold-Maxwell $U$, French BW, Meinke LJ. Susceptibility of Nebraska Western corn rootworm (Coleoptera: Chrysomelidae) populations to Bt corn events. J Econ Entomol. 2015;108(2):742-51.

18. Gundersen-Rindal D, Adrianos S, Allen MM, Becnel J, Chen Y, Choi M, Estep A, Evans J, Garczynski S, Geib S. Arthropod genomics research in the United States Department of Agriculture-Agricultural Research Service: applications of RNA interference and CRISPR gene editing technologies in pest control. Trends Entomol. 2017;13:109-37.

19. Huvenne H, Smagghe G. Mechanisms of dsRNA uptake in insects and potential of RNAi for pest control: a review. J Insect Physiol. 2010;56(3):227-35.

20. Baum JA, Bogaert T, Clinton W, Heck GR, Feldmann P, llagan O, Johnson S, Plaetinck G, Munyikwa T, Pleau M, et al. Control of coleopteran insect pests through RNA interference. Nat Biotechnol. 2007;25(11):1322-6.

21. Li H, Khajuria C, Rangasamy M, Gandra P, Fitter M, Geng C, Woosely A, Hasler J, Schulenberg $\mathrm{G}$, Worden $\mathrm{S}$, et al. Long dsRNA but not siRNA initiates RNAi in western corn rootworm larvae and adults. J Appl Entomol. 2015;139(6):432-45.

22. Rangasamy M, Siegfried BD. Validation of RNA interference in western corn rootworm Diabrotica virgifera virgifera LeConte (Coleoptera: Chrysomelidae) adults. Pest Manag Sci. 2012;68(4):587-91.

23. Fishilevich E, Velez AM, Storer NP, Li HR, Bowling AJ, Rangasamy M, Worden SE, Narva KE, Siegfried BD. RNAi as a management tool for the western corn rootworm, Diabrotica virgifera virgifera. Pest Manag Sci. 2016;72(9):1652-63.

24. Higgins CF. ABC transporters - from microorganisms to man. Annu Rev Cell Biol. 1992:8:67-113.

25. Dermauw W, Van Leeuwen $T$. The ABC gene family in arthropods: comparative genomics and role in insecticide transport and resistance. Insect Biochem Molec. 2014;45:89-110.

26. George AM, Jones PM. Perspectives on the structure-function of ABC transporters: the switch and Constant contact models. Prog Biophys Mol Bio. 2012;109(3):95-107.

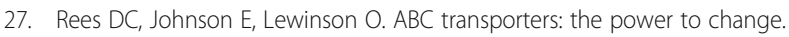
Nat Rev Mol Cell Bio. 2009;10(3):218-27.

28. Dean M, Hamon Y, Chimini G. The human ATP-binding cassette (ABC) transporter superfamily. J Lipid Res. 2001;42(7):1007-17.

29. Dean M, Annilo T. Evolution of the ATP-binding cassette (ABC) transporter superfamily in vertebrates. Annu Rev Genom Hum G. 2005;6:123-42.

30. Broehan $G$, Kroeger $T$, Lorenzen M, Merzendorfer $H$. Functional analysis of the ATP-binding cassette (ABC) transporter gene family of Tribolium castaneum. BMC Genomics. 2013;14:6.

31. Ewart GD, Cannell D, Cox GB, Howells AJ. Mutational analysis of the traffic Atpase $(A B C)$ transporters involved in uptake of eye pigment precursors in Drosophila melanogaster - implications for structure-function-relationships. J Biol Chem. 1994;269(14):10370-7.

32. Grubbs N, Haas S, Beeman RW, Lorenzen MD. The ABCs of Eye Color in Tribolium castaneum: Orthologs of the Drosophila white, scarlet, and brown Genes. Genetics. 2015;199(3):749.

33. Liu SM, Zhou S, Tian L, Guo EN, Luan YX, Zhang JZ, Li S. Genome-wide identification and characterization of ATP-binding cassette transporters in the silkworm, Bombyx mori. BMC Genomics. 2011;12:491.

34. Mackenzie SM, Brooker MR, Gill TR, Cox GB, Howells AJ, Ewart GD. Mutations in the white gene of Drosophila melanogaster affecting $A B C$ transporters that determine eye colouration. Biochim Biophys Acta. 1999;1419(2):173-85.

35. Aurade RM, Jayalakshmi SK, Sreeramulu K. P-glycoprotein ATPase from the resistant pest, Helicoverpa armigera: purification, characterization and effect of various insecticides on its transport function. Biochim Biophys Acta. 2010; 1798(6):1135-43.

36. Buss DS, Callaghan A. Interaction of pesticides with p-glycoprotein and other ABC proteins: a survey of the possible importance to insecticide, herbicide and fungicide resistance. Pestic Biochem Phys. 2008;90(3):141-53.

37. Coates BS. Bacillus thuringiensis toxin resistance mechanisms among Lepidoptera: progress on genomic approaches to uncover causal mutations in the European corn borer, Ostrinia nubilalis. Curr Opin Insect Sci. 2016;15:70-7.

38. Pauchet Y, Bretschneider A, Augustin S, Heckel DG. A P-Glycoprotein Is Linked to Resistance to the Bacillus thuringiensis Cry3Aa Toxin in a Leaf Beetle. Toxins. 2016;8(12):362.

39. Flagel LE, Swarup S, Chen M, Bauer C, Wanjugi H, Carroll M, Hill P, Tuscan M, Bansal R, Flannagan R, et al. Genetic Markers for Western Corn Rootworm Resistance to Bt Toxin. G3. 2015;5(3):399-405.

40. Chu CC, Zavala JA, Spencer JL, Curzi MJ, Fields CJ, Drnevich J, Siegfried BD, Seufferheld MJ. Patterns of differential gene expression in adult rotationresistant and wild-type western corn rootworm digestive tracts. Evol Appl. 2015;8(7):692-704.

41. Eyun SI, Wang HC, Pauchet Y, Ffrench-Constant RH, Benson AK, Valencia-Jimenez A, Moriyama EN, Siegfried BD. Molecular Evolution of Glycoside Hydrolase Genes in the Western Corn Rootworm (Diabrotica virgifera virgifera). PLoS One. 2014;9(4):e94052.

42. Flagel LE, Bansal R, Kerstetter RA, Chen M, Carroll M, Flannagan R, Clark T, Goldman BS, Michel AP. Western corn rootworm (Diabrotica virgifera virgifera) transcriptome assembly and genomic analysis of population structure. BMC Genomics. 2014;15:195.

43. Siegfried BD, Waterfield N, ffrench-Constant RH. Expressed sequence tags from Diabrotica virgifera virgifera midgut identify a coleopteran cadherin and a diversity of cathepsins. Insect Mol Biol. 2005;14(2): $137-43$.

44. Coates BS, Alves AP, Wang HC, Walden KKO, French BW, Miller NJ, Abel CA, Robertson HM, Sappington TW, Siegfried BD. Distribution of Genes and Repetitive Elements in the Diabrotica virgifera virgifera Genome Estimated Using BAC Sequencing. J Biomed Biotechnol. 2012;2012:604076.

45. Wang YB, Zhang H, Li HC, Miao XX. Second-Generation Sequencing Supply an Effective Way to Screen RNAi Targets in Large Scale for Potential Application in Pest Insect Control. PLoS One. 2011;6(4):e18644.

46. Grubbs N, Chu F-C, Lorenzen M. Window to the Fluorescence: The White Eye-Color Gene of Western Corn Rootworm, Diabrotica virgifera virgifera. bioRxiv. 2019:552935.

47. Labbe R, Caveney S, Donly C. Genetic analysis of the xenobiotic resistance-associated $A B C$ gene subfamilies of the Lepidoptera. Insect Mol Biol. 2011;20(2):243-56.

48. Lage H. ABC-transporters: implications on drug resistance from microorganisms to human cancers. Int J Antimicrob Agents. 2003;22(3):188-99.

49. Merzendorfer $\mathrm{H}$. ABC transporters and their role in protecting insects from pesticides and their metabolites. Adv Insect Physiol. 2014;46:1-72.

50. Tian LX, Song TX, He RJ, Zeng Y, Xie W, Wu QJ, Wang SL, Zhou XG, Zhang YJ. Genome-wide analysis of ATP-binding cassette (ABC) transporters in the sweetpotato whitefly, Bemisia tabaci. BMC Genomics. 2017;18:330.

51. Heckel DG. Roles of $A B C$ proteins in the mechanism and Management of $B t$ Resistance. Cabi Biotech Ser. 2015;4:98-106.

52. Sato R, Adegawa S, Li X, Tanaka S, Endo H. Function and Role of ATPBinding Cassette Transporters as Receptors for 3D-Cry Toxins. Toxins (Basel). 2019;11(2):E124

53. Gahan LJ, Pauchet $Y$, Vogel H, Heckel DG. An ABC Transporter Mutation Is Correlated with Insect Resistance to Bacillus thuringiensis Cry1Ac Toxin. PLoS Genet. 2010;6(12):e1001248.

54. Xiao Y, Zhang T, Liu C, Heckel DG, Li X, Tabashnik BE, Wu K. Mis-splicing of the $A B C C 2$ gene linked with Bt toxin resistance in Helicoverpa armigera. Sci Rep. 2014;4:6184.

55. Atsumi S, Miyamoto K, Yamamoto K, Narukawa J, Kawai S, Sezutsu H, Kobayashi I, Uchino K, Tamura T, Mita K, et al. Single amino acid mutation in an ATP-binding cassette transporter gene causes resistance to Bt toxin Cry1Ab in the silkworm, Bombyx mori. Proc Natl Acad Sci U S A. 2012;109(25):E1591-8.

56. Park Y, Gonzalez-Martinez RM, Navarro-Cerrillo G, Chakroun M, Kim Y, Ziarsolo P, Blanca J, Canizares J, Ferre J, Herrero S. ABCC transporters mediate insect resistance to multiple Bt toxins revealed by bulk segregant analysis. BMC Biol. 2014;12:46. 
57. Stevens T, Song SS, Bruning JB, Choo A, Baxter SW. Expressing a moth abcc2 gene in transgenic Drosophila causes susceptibility to Bt Cry1Ac without requiring a cadherin-like protein receptor. Insect Biochem Mol Biol. 2017;80:61-70.

58. Coates BS, Siegfried BD. Linkage of an ABCC transporter to a single QTL that controls Ostrinia nubilalis larval resistance to the Bacillus thuringiensis Cry1 Fa toxin. Insect Biochem Mol Biol. 2015;63:86-96.

59. Flagel L, Lee YW, Wanjugi H, Swarup S, Brown A, Wang JL, Kraft E, Greenplate J, Simmons J, Adams N, et al. Mutational disruption of the $A B C C 2$ gene in fall armyworm, Spodoptera frugiperda, confers resistance to the Cry1Fa and Cry1A.105 insecticidal proteins. Sci Rep. 2018;8:7255.

60. Tay WT, Mahon RJ, Heckel DG, Walsh TK, Downes S, James WJ, Lee SF, Reineke A, Williams AK, Gordon KHJ. Insect resistance to Bacillus thuringiensis toxin Cry2Ab is conferred by butations in an $A B C$ transporter subfamily A protein. PLoS Genet. 2015;11(11):e1005534.

61. Wang J, Wang H, Liu S, Liu L, Tay WT, Walsh TK, Yang Y, Wu Y. CRISPR/Cas9 mediated genome editing of Helicoverpa armigera with mutations of an $A B C$ transporter gene $\mathrm{HaABCA} 2$ confers resistance to Bacillus thuringiensis Cry2A toxins. Insect Biochem Mol Biol. 2017:87:147-53.

62. Guo ZJ, Kang S, Zhu X, Xia JX, Wu QJ, Wang SL, Xie W, Zhang YJ. Downregulation of a novel $A B C$ transporter gene (Pxwhite) is associated with Cry 1 Ac resistance in the diamondback moth, Plutella xylostella (L.). Insect Biochem Mol Biol. 2015;59:30-40.

63. Zhang TT, Coates BS, Wang YQ, Wang YD, Bai SX, Wang ZY, He KL. Downregulation of aminopeptidase $N$ and $A B C$ transporter subfamily $G$ transcripts in Cry $1 \mathrm{Ab}$ and Cry1Ac resistant Asian corn borer, Ostrinia furnacalis (Lepidoptera: Crambidae). Int J Biol Sci. 2017;13(7):835-51.

64. Evans JD, McKenna D, Scully E, Cook SC, Dainat B, Egekwu N, Grubbs N, Lopez D, Lorenzen MD, Reyna SM. Genome of the small hive beetle (Aethina tumida, Coleoptera: Nitidulidae), a worldwide parasite of social bee colonies, provides insights into detoxification and herbivory. GigaScience. 2018;7(12):giy138.

65. Sturm A, Cunningham P, Dean M. The ABC transporter gene family of Daphnia pulex. BMC Genomics. 2009:10:170.

66. Dermauw W, Osborne EJ, Clark RM, Grbić M, Tirry L, Van Leeuwen T. A burst of $A B C$ genes in the genome of the polyphagous spider mite Tetranychus urticae. BMC Genomics. 2013;14(1):317.

67. Quazi F, Molday RS. Lipid transport by mammalian ABC proteins. Essays Biochem. 2011;50:265-90.

68. Krishnamurthy PC, Du GQ, Fukuda Y, Sun DX, Sampath J, Mercer KE, Wang JF, Sosa-Pineda B, Murti KG, Schuetz JD. Identification of a mammalian mitochondrial porphyrin transporter. Nature. 2006;443(7111):586-9.

69. Pondarre C, Antiochos BB, Campagna DR, Greer EL, Deck KM, McDonald A, Han AP, Medlock A, Kutok JL, Anderson SA, et al. The mitochondrial ATPbinding cassette transporter $A b c b 7$ is essential in mice and participates in cytosolic iron-sulfur cluster biogenesis. Hum Mol Genet. 2006;15(6):953-64.

70. Barthelme D, Dinkelaker S, Albers SV, Londei P, Ermler U, Tampe R. Ribosome recycling depends on a mechanistic link between the FeS cluster domain and a conformational switch of the twin-ATPase ABCE1. Proc Natl Acad Sci U S A. 2011;108(8):3228-33.

71. Paytubi S, Wang XM, Lam YW, Izquierdo L, Hunter MJ, Jan E, Hundal HS, Proud CG. ABC50 promotes translation initiation in mammalian cells. J Biol Chem. 2009;284(36):24061-73.

72. Sullivan DT, Grillo SL, Kitos RJ. Subcellular localization of the first three enzymes of the ommochrome synthetic pathway in Drosophila melanogaster. J Exp Zool. 1974;188(2):225-33.

73. Tarr PT, Tarling EJ, Bojanic DD, Edwards PA, Baldan A. Emerging new paradigms for ABCG transporters. Biochim Biophys Acta. 2009;1791 (7):584-93.

74. Lorenzen MD, Brown SJ, Denell RE, Beeman RW. Cloning and characterization of the Tribolium castaneum eye-color genes encoding tryptophan oxygenase and kynurenine 3-monooxygenase. Genetics. 2002; 160(1):225-34.

75. Chu F, Klobasa W, Wu P, Pinzi S, Grubbs N, Gorski S, Cardoza Y, Lorenzen MD. Germline transformation of the western corn rootworm, Diabrotica virgifera virgifera. Insect Mol Biol. 2017;26(4):440-52.

76. Andrews S: FastQC: a quality control tool for high throughput sequence data. http://www.bioinformatics.babraham.ac.uk/projects/fastqc/; 2010.

77. Bolger AM, Lohse $\mathrm{M}$, Usadel B. Trimmomatic: a flexible trimmer for Illumina sequence data. Bioinformatics. 2014;30(15):2114-20.

78. Xie YL, Wu GX, Tang JB, Luo RB, Patterson J, Liu SL, Huang WH, He GZ, Gu SC, Li SK, et al. SOAPdenovo-trans: de novo transcriptome assembly with short RNA-Seq reads. Bioinformatics. 2014;30(12):1660-6.
79. Grabherr MG, Haas BJ, Yassour M, Levin JZ, Thompson DA, Amit I, Adiconis X, Fan L, Raychowdhury R, Zeng QD, et al. Full-length transcriptome assembly from RNA-Seq data without a reference genome. Nat Biotechnol. 2011;29(7):644-U130.

80. Li WZ, Godzik A. Cd-hit: a fast program for clustering and comparing large sets of protein or nucleotide sequences. Bioinformatics. 2006;22(13):1658-9.

81. Waterhouse RM, Tegenfeldt F, Li J, Zdobnov EM, Kriventseva EV. OrthoDB: a hierarchical catalog of animal, fungal and bacterial orthologs. Nucleic Acids Res. 2013;41(D1):D358-65.

82. Simao FA, Waterhouse RM, loannidis P, Kriventseva EV, Zdobnov EM. BUSCO: assessing genome assembly and annotation completeness with single-copy orthologs. Bioinformatics. 2015;31(19):3210-2.

83. Haas B, Papanicolaou A: TransDecoder (find coding regions within transcripts). https://github.com//ransDecoder/TransDecoder/wiki; 2016.

84. Conesa A, Gotz S, Garcia-Gomez JM, Terol J, Talon M, Robles M. Blast2GO: a universal tool for annotation, visualization and analysis in functional genomics research. Bioinformatics. 2005;21(18):3674-6.

85. Gotz S, Garcia-Gomez JM, Terol J, Williams TD, Nagaraj SH, Nueda MJ, Robles M, Talon M, Dopazo J, Conesa A. High-throughput functional annotation and data mining with the Blast2GO suite. Nucleic Acids Res. 2008:36(10):3420-35.

86. Altschul SF, Madden TL, Schaffer AA, Zhang J, Zhang Z, Miller W, Lipman DJ. Gapped BLAST and PSI-BLAST: a new generation of protein database search programs. Nucleic Acids Res. 1997;25(17):3389-402.

87. Marchler-Bauer A, Bo Y, Han LY, He JE, Lanczycki CJ, Lu SN, Chitsaz F, Derbyshire MK, Geer RC, Gonzales NR, et al. CDD/SPARCLE: functional classification of proteins via subfamily domain architectures. Nucleic Acids Res. 2017;45(D1):D200-3.

88. Kumar S, Stecher G, Li M, Knyaz C, Tamura K. MEGA X: molecular evolutionary genetics analysis across computing platforms. Mol Biol Evol. 2018;35(6):1547-9.

89. Hall BG. Building phylogenetic trees from molecular data with MEGA. Mol Biol Evol. 2013:30(5):1229-35.

\section{Publisher's Note}

Springer Nature remains neutral with regard to jurisdictional claims in published maps and institutional affiliations.

\section{Ready to submit your research? Choose BMC and benefit from:}

- fast, convenient online submission

- thorough peer review by experienced researchers in your field

- rapid publication on acceptance

- support for research data, including large and complex data types

- gold Open Access which fosters wider collaboration and increased citations

- maximum visibility for your research: over $100 \mathrm{M}$ website views per year

At $\mathrm{BMC}$, research is always in progress.

Learn more biomedcentral.com/submissions 